analysis was performed using SPSS software version 25 for Windows (SPSS Inc., Chicago, IL, USA) (Corporation, 2017). Results From 281 patients, 60.14\% and 38.14\% patients were presented for ERCP procedures before and after COVID-19 period respectively. Higher proportion of patients was presented with liver dysfunction before pandemic period as compared to pandemic period $(\mathrm{P}<0.005)$ and use of antibiotics was significantly higher in pandemic period $(\mathrm{P}<0.05)$. the success rate of ERCP procedure was higher before emergence of the pandemic and lesser during the COVID-19 first wave as $86.39 \%$ and $77.67 \%$ respectively but the results were insignificant $(\mathrm{P}=0.07)$. A statistically significant but negative correlation was observed between cholangitis and stent insertion with ERCP success and positive correlation between sphincterotomy and ERCP success as $(\mathrm{r}=-0.129, \quad \mathrm{P}=0.030), \quad(\mathrm{r}=-0.172$, $\mathrm{P}=0.004)$ and $(\mathrm{r}=0.232, \mathrm{P}<0.001)$ respectively. In binary logistic regression analysis, sphincterotomy $(\beta=2.800$, $\mathrm{P}=0.028)$ and stent insertion $(\beta=0.852, \mathrm{P}=0.046)$ were statistically significant predictors of ERCP outcomes. There was no statistically significant impact of cholangitis in the success of ERCP $(\beta=1.672, \mathrm{P}=0.109)$.

Conclusion COVID-19 pandemic significantly reduced ERCP procedures and success rate was also lowered due to restriction on endoscopic services in UK and all over the world. The endoscopic services can be resumed subject to specific SOPs to be followed in endoscopic units without affecting the safety of staff and patients.

\section{P037 TRANSAMINITIS AND IRRITABLE BOWEL SYNDROME (IBS): A COMPREHENSIVE REVIEW}

${ }^{1}$ Eyad Gadour*, ${ }^{2}$ Zeinab Hassan. ${ }^{1}$ University Hospitals of Morecambe Bay NHS Foundation Trust, UKi ${ }^{2}$ Stockport Hospital NHS Foundation Trust, Manchester, UK

\subsection{6/gutjnl-2021-BASL.46}

Introduction We observed in literature that irritable bowel syndrome (IBS) may be linked with irregular parameters of metabolic system (MS) and liver function. For that reason, we are conducting this systematic review to comprehensively analyze the association of transaminitis (elevated ALT) with IBS.

Methodology This systematic review was designed by following methods described in the Cochrane Handbook for Systematic Reviews of Interventions. Published peer-reviewed journal articles were included. Data was extracted based on study designs Age, gender, author, date of publication or availability online, publication type, participants, gender $(\mathrm{M} / \mathrm{F})$ and types of IBS.

Results Our electronic of multiple databases yielded a total of 519 preliminary studies; we then removed duplicate studies and left with 326 studies. After reviewing full text of these articles, a total of 83 studies were eliminated and lastly, three studies were selected for this systematic review for quantitative and qualitative analysis. All the enrolled subjects in included studies were diagnosed with IBS by Rome II and III criteria and among these sub-jects, 50.4\% had IBS-D, $13.8 \%$ had IBS-C, 30.3\% had IBS-M, and 3.5\% had IBS-U. The prevalence of elevated alanine aminotransferase (ALT) with other liver enzymes $(\gamma$-GT levels and AST) in patients with irritable bowel syndrome whether their BMI were high or not $(16.9 \%$ vs. $7.7 \% ; \mathrm{p}=0.015)$ and $\gamma$-GT $(24.1 \%$ vs. $11.5 \% ; \mathrm{p}=0.037)$ Lee et al., 2016. ${ }^{1}$ The IBS-D subtype was seen more commonly in patients whose alcohol intake were significantly high however their study data showed no significant change in elevation of ALT. The upper limits normal values for serum liver enzymes were de $\neg$ fined as forty-one international per liter in males and thirty-one international unit per liter in females for ALT. No significant relationships were observed between IBS status and elevated $\gamma$-GT (OR, 1.647; 95\% CI, 0.784-3.461). ${ }^{1}$ Conclusion The review study proposes a potential relation between elevated ALT levels, MS, and IBS, and this review might be the first review in IBS patients to observe the association of elevated ALT in IBS population. Although further additional trials with a large sample size will be require confirming these results. Furthermore, for assessing the efficacy of the manipulation of gut microbiota ran $\neg$ domized controlled trials in a large population of IBS patients are needed to establish a causal-resultant relationship IBS and MS and liver damage.

\section{REFERENCES}

1. Lee SH, Kim KN, Kim KM, Joo NS. Irritable bowel syndrome may be associated with elevated alanine aminotransferase and metabolic syndrome. Yonsei Med J 2016;57(1):146-152.

2. Khayyatzadeh SS, Esmaillzadeh A, Saneei P, Keshteli AH, Adibi P. Dietary patterns and prevalence of irritable bowel syndrome in Iranian adults. Neurogastroenterol Motil 2016;28(12):1921-1933. doi:10.1111/nmo.12895

\section{P038 THE METABOLIC SWITCH OF HEPATITIS C VIRUS- RELATED HEPATOCELLULAR CARCINOMA AFTER EPHRIN TYPE-A RECEPTOR 2 IS KNOCKED DOWN}

\begin{abstract}
1,2Zijian Zhang* ${ }^{3}$ Qi Zhou. 'Department of General Surgery, The Seventh Affiliated Hospital, Sun Yat-sen University, Shenzhen, China; ' Digestive Medine Center, The Seventh Affiliated Hospital, Sun Yat-sen University, Shenzhen, China; ${ }^{3}$ Department of General Surgery, Huiya Hospital of the First Affiliated Hospital of Sun-Yat-sen University, Huizhou, China
\end{abstract}

\subsection{6/gutjnl-2021-BASL.47}

Purpose Hepatitis C virus (HCV) is a major pathogen of liver diseases, including hepatitis, liver cirrhosis, and hepatocellular carcinoma. Few strategies were applied in the prevention and treatment of Hepatitis C. More and more evidence shows that ephrin receptor A2 (EphA2) is a key factor in the HCV entrance into the liver cells. During our study of metabolic reconstruction of liver cancer cells, we found metabolic progress changed after knocking down the expression of EphA2. That means EphA2 participates not only in the migration, integrin-mediated adhesion, proliferation, and differentiation but also in the metabolic biological processes. We designed an EphA2+metabolic classifier that can distinguish different subtypes of HCV-related HCC which have different characteristics, prognoses, and treatments.

Method Utilizing the data from gene expression omnibus (GEO) and normal liver tissues of GTEx, we focus on the metabolic changes between wild-type $\mathrm{HuH} 7$ cells and EphA2knocked down HuH7 cells. We ran the Gene set enrichment analysis (GSEA) and Gene set variation analysis (GSVA) on the differential expression genes (DEGs) calculated by several packages of $\mathrm{R}$ language. And we tried to find the different expression patterns in gene set levels, based on the metabolism-related gene sets. Data from HCV-related HCC in GEO were explored. Bioinformatics methods are used to analyze the genomics, transcriptomics, and clinical data.

Results After utilizing the data from HCV-related HCC of GEO and normal liver tissues of GTEx, 101 LLPS related genes were selected for further research. We ran the GSEA and GSVA, and we found three significantly changed gene sets 